\title{
Singularity of the Density of States for One-Dimensional Chains with Random Couplings
}

\author{
Massimo Campanino ${ }^{1 \star}$ and J. Fernando Perez ${ }^{2 \star \star}$ \\ ${ }^{1}$ Instituto di Mathematica, Departimento di Matematica Università di Bologna, piorza Porta S. \\ Donato, 5, I-40127 Bologna, Italy \\ ${ }^{2}$ Departimento de Fisica Matematica, IFUSP, P.O. Box 20516, São Paulo, Brazil
}

\begin{abstract}
We prove that the density of states for the tight-binding model with off-diagonal disorder under general conditions diverges for $R \rightarrow 0$ at least as $\sim \frac{1}{|E|(\ln |E|)^{4}}$. This result is established through the study of the recurrence properties of an associated Markov chain.
\end{abstract}

\section{Introduction}

In the field of Schrödinger equations with random potential (see [S] and references therein) a great deal of attention has been given to the study of the properties of the density of states as a function of the energy $E$. For lattice systems with diagonal disorder Wegner ([W]) proved that if the distribution of the potential is absolutely continuous with bounded density, then the integrated density of states is absolutely continuous and its derivative $\rho(E)$ is bounded. With more detailed assumptions and/or in one dimension one can prove smoothness properties of the density of states a) in any dimension at high disorder ([CFS, BCKP]); b) in one dimension at any disorder by using transfer matrix methods ([ST, CK $]$ ). In $[\mathrm{CK}]$ it is proven that for a wide class of distributions for the potential including non-absolutely continuous ones the density of states is smooth in one-dimension.

The case with only off-diagonal disorder, i.e. when only the couplings between different sites are random, presents some different features. Here Wegner's result does not apply. Indeed Dyson ([Dy]) found a one-dimensional model whose density of states can be exactly computed and displays a singularity of the type $\frac{1}{|E|(\ln |E|)^{3}}$ as $E \rightarrow 0$. It has been argued that this singularity should not be particular to this model. In [ER] and [TC] numerical computations and heuristic arguments are presented to support this claim.

\footnotetext{
* Partial financial support by GNAFA (CNR)

$\star \star$ Partial financial support by CNPq, grant n.303795-77FA
} 
Here we prove a lower bound $\frac{\text { const. }}{|E|(\ln |E|)^{4}}$ for the density of states as $E \rightarrow 0$. We allow a general distribution for the potential absolutely continuous with continuous density and with support bounded away from the origin.

Here is a sketch of our argument. We express the expected density of states in a finite volume in terms of the distribution of a Markov chain at a finite time. We study then the invariant density of the Markov chain. For $E$ sufficiently small the invariant density can be estimated in some $E$-dependent interval by studying the recurrence properties of the Markov chain. Here we make use of methods introduced for Markov chains with continuous state space by Athreya and Ney ([AN]). As the densities at finite times approach for large times (that correspond to large volumes for the physical system) the invariant density, we can use the bound to get our result.

In the following, when no confusion can arise, we shall use the same letters $c$, $\bar{c}$ etc. to indicate possibly different constants.

\section{Description of the Model and Sketch of the Proof}

Let $H$ be the operator on $l^{2}(\mathbf{Z})$,

$$
H u(n)=W_{n, n+1} u(n+1)+W_{n-1, n} u(n-1),
$$

where $W_{n, n+1}$ are i.i.d. distributed random variables. We shall put $W_{n, n+1}=V_{n+1}$ for $n \geqq 0$ and $W_{n, n+1}=V_{n}$ for $n \leqq 1$. We assume that the common distribution of these variables has support in some interval $[\bar{\alpha}, \bar{\beta}]$ with $0<\bar{\alpha}<\bar{\beta}$ and continuous density $f$.

Let $\Lambda_{N}$ be the interval $[-N, N]$ in $\mathbf{Z}$. We shall denote by $H_{N}$ the operator $H$ restricted to $l^{2}\left(\Lambda_{N}\right)$ with boundary conditions $u(x)=0$ for $x \notin \Lambda_{N}$.

We can compute $G_{N}(0,0 ; z) \equiv\left\langle 0\left|\left(H_{N}-z\right)^{-1}\right| 0\right\rangle$ by observing that $G_{N}(0,0 ; z)=$ $g(0)$, where $g$ satisfies the equation

$$
\left(H_{N}-z\right) g=\delta_{0}
$$

with boundary conditions $g(N+1)=g(-N-1)=0$. If we define $\Delta_{+, k}(z) \Delta_{-, k}(z)$ for $0 \leqq k \leqq N$ by

$$
\Delta_{+, k}(z)=V_{k+1} \frac{g(k+1)}{g(k)}, \quad \Delta_{-, k}(z)=V_{-k-1} \frac{g(-k-1)}{g(-k)},
$$

and insert them in Eq. (2.2), we see that they satisfy the equations

$$
\begin{aligned}
& \Delta_{+, k-1}(z)=\frac{V_{k}^{2}}{z-\Delta_{+, k}(z)}, \\
& \Delta_{-, k-1}(z)=\frac{V_{-k}^{2}}{z-\Delta_{-, k}(z)},
\end{aligned}
$$

with initial conditions $\Delta_{+, N}(z)=\Delta_{-, N}(z)=0$ and that

$$
g(0)=G_{N}(0,0 ; z)=\frac{1}{\Delta_{+, 0}(z)+\Delta_{-, 0}(z)-z} .
$$


Notice that $\Delta_{+, 0}(z), \Delta_{-, 0}(z)$ are holomorphic functions of $z$, that are real for real $z$ and have a negative imaginary part if $z$ has a positive imaginary part. Therefore, in the distributional sense,

$$
\lim _{\varepsilon \downarrow 0} \operatorname{Im} \frac{1}{\left[\Delta_{+, 0}(E+i \varepsilon)+\Delta_{-, 0}(E+i \varepsilon)-E+i \varepsilon\right]}=-\pi \delta\left(\Delta_{+, 0}(E)+\Delta_{-, 0}(E)-E\right) .
$$

It follows that the averaged density of states in the finite volume $[-N, N]$ is given by

$$
\begin{aligned}
\rho_{N}(E) & =\lim _{\varepsilon \downarrow 0} \frac{1}{\pi} \operatorname{Im} \mathbb{E}\left(\frac{1}{\left[\Delta_{+, 0}(E+i \varepsilon)+\Delta_{-, 0}(E+\mathrm{i} \varepsilon)-E+\mathrm{i} \varepsilon\right]}\right) \\
& =\int q_{E}^{(N)}(E-x) q_{E}^{(N)}(x) d x,
\end{aligned}
$$

where $q_{E}^{(N)}(x)$ is the common density of the two independent random variables $\Delta_{+, 0}(E)$ and $\Delta_{-, 0}(E)$.

We are thus led to consider the random process defined by (2.4) for real $z$. It will prove convenient to introduce the logarithms of $\Delta_{+, k} \equiv \Delta_{k}$. In order to take account of the negative values and to have a bijection between the two variables, we introduce the map

$$
\mathbb{R} \backslash\{0\} \rightarrow \mathbb{R} \times\{-1,1\}, \Delta \mapsto(\ln |\Delta|, \operatorname{sgn} \Delta)=(u, \varepsilon) .
$$

In terms of the new variables the process (2.4) is described by the equations

$$
u_{k-1}=\ln V_{k}^{2}-\ln \left|E-\varepsilon_{k} e^{u_{k}}\right|, \quad \varepsilon_{k-1}=\left\{\begin{aligned}
1, & \text { if } \quad E>\varepsilon_{k} e^{u_{k}} \\
-1, & \text { if } \quad E<\varepsilon_{k} e^{u_{k}}
\end{aligned}\right.
$$

The sequence $\left\{\Delta_{k}\right\} k=-N, \ldots, 0$ defines a Markov chain with $\mathbb{R}$ as a state space. We will consider this Markov chain for $E$ sufficiently small and we will prove that this Markov chain approaches the stationary measure exponentially fast and uniformly for $E$ bounded away from 0 . We then estimate $q_{E}(x)$ from below by using the connection between the invariant measure of a set and the expectation of the return time to it (see $[\mathrm{C}, \mathrm{AN}]$ ) to obtain the lower bound $q_{E}(x) \geqq$ const. $\frac{1}{|x|(\ln |E|)}$ for $b E \leqq x \leqq \frac{1}{b E}$ and $b$ a sufficiently large constant independent from $E$. The result will follow from this bound, the equality (2.13) together with convergence of $q_{E}^{(N)}(x)$ to $q_{E}(x)$.

\section{The Invariant Measure and the Approach to Equilibrium}

The equation (2.9) defines a Markov chain $\left\{x^{(n)}, n \geqq 0\right\}$ (we reverse here the time direction in order to follow the usual conventions). The state space $\mathscr{S}=\mathbb{R} \times\{-1,1\}$ can be endowed with the natural measure $v$ that, restricted to each of the two copies of $\mathbb{R}$, is equal to the Lebesgue measure. The transition probability density of the Markov chain with respect to $v$ will be denoted by $p_{E}(\xi, \eta)$, and for any Borel set $A \subset \mathscr{S}$ we define $P_{E}(\xi, A)=\int_{A} p_{E}(\xi, \eta) d \eta$; the subscript $E$ in $p_{E}$ and $P_{E}$ will be omitted when no confusion can arise. $p_{E}^{(n)}$ and $P_{E}^{(n)}$ will denote respectively the 
$n$-step transition probability density and the $n$-step transition kernel of the Markov chain.

The statements of the following three lemmas contain the information that we need on the invariant measure of our Markov chain. The proofs of the lemmas are deferred to the Appendix.

For $b<1$ and $\delta>0$ we define $J_{b}=[\ln b,-\ln b]$ and $\mathscr{I}_{b, \delta}$ as the set of the intervals $I$ such that $I \subset J_{b}$ and the length of $I$ is equal to $\delta$.

3.1. Lemma. There exists $E_{0}>0, b>0, \delta>0$ and $c>0$ such that for $0<E<E_{0}$ and $I \in \mathscr{I}_{b E, \delta}$,

$$
p_{E}^{(2)}(\xi, \eta) \geqq c \quad \text { for } \quad \xi, \eta \in I \times\{1\} .
$$

3.2. Lemma. There exists $E_{0}>0$ and $b>0$ such that for $0<E<E_{0}$ there is $n_{0}$ positive integer such that

$$
\inf _{\xi \in \mathscr{S}_{\eta \in J_{b} \times\{1\}}} p_{E}^{\left(n_{0}\right)}(\xi, \eta) \geqq c>0,
$$

where $n_{0}$ and $c$ can be chosen uniformly in $E$ for $E$ bounded away from 0 .

Given a closed interval $I \subset \mathbb{R}$ we define $T_{I}$ as the time of first arrival of the Markov chain to the set $I \times\{1\}$, i.e.

$$
T_{I}=\inf \left\{n \geqq 1 \mid x^{(n)} \in I \times\{1\} .\right.
$$

3.3. Lemma. There exists $b>0, \delta>0, E_{0}>0$ and $c>0$ such that for $0<E<E_{0}$ and $I \in \mathscr{I}_{b E, \delta}$,

$$
\sup _{\xi \in I \times\{1\}} \mathbb{E}_{\xi}\left(T_{I}\right) \leqq c(\ln |E|)^{2},
$$

where $\mathbb{E}_{\xi}$ denotes the expectation with respect to the Markov chain with transition kernel $P_{E}$ and initial condition $\xi$.

From Lemma 3.2 it follows that for the considered values of $E$ there exists a unique invariant measure $Q_{E}$ with density $q_{E}(x)$ which is approached exponentially fast in the variation distance uniformly for $E$ bounded away from 0 , i.e.

$$
\sup _{\xi, A} \mid P_{E}^{(n)}(\xi, A)-Q_{E}(A) \leqq c \rho^{n}
$$

with $c>0$ and $0<\rho<1$ (see [D] Chap. V, Sect. 5, case b). Moreover Lemma 3.2 shows that every $I \in \mathscr{I}_{b E, \delta}$ is a recurrent set for the Markov chain with kernel $P_{E}$. This together with Lemma 3.1 allows us to use the construction by Athreya and Ney of an auxiliary chain with a recurrent state (see in [AN] the regeneration lemma and Sect. 5). In the notation of [AN] if $I \in \mathscr{I}_{b E, \delta}$ with $b$ and $\delta$ given by Lemma $3.1,\left\{x^{(n)}, n \geqq 0\right\}$ is a $(I, \lambda, \phi, 2)$-recurrent Markov chain where $\lambda=\inf _{\xi, \eta \in I \times\{1\}} p_{E}^{(2)}(\xi, \eta)$ and $\phi=\frac{v}{|I|}$ with $v$ equal to the Lebesgue measure.

Let now $M_{I}=\sup _{\xi \in I \times\{1\}} \mathbb{E}_{\xi}\left(T_{I}\right)$. If we perform the construction of the equivalent Markov chain as in [AN], Sect. 5 with a state $\Theta$ corresponding to the interval $I$, and call $\widetilde{Q}$ and $\widetilde{\mathbb{E}}$ the invariant measure and the expectation for the equivalent 
chain, we have

and

$$
\tilde{\mathbb{E}}_{\Theta}\left(\widetilde{T}_{\Theta}\right) \leqq M_{I} \lambda^{-1}
$$

$$
\tilde{Q}(\Theta)=\lambda Q(I),
$$

where $\widetilde{T}_{\Theta}$ is the return time to the state $\Theta$ in the equivalent chain.

Formula (3.5) follows from the fact that

$$
\tilde{T}_{\Theta}=T_{I}+\sum_{j \geqq 2} \chi\left(\delta_{T_{I}^{(k)}}=0, \text { for } 1 \leqq k \leqq j-1\right)\left(T_{I}^{(j)}-T_{I}^{(j-1)}\right),
$$

where $\chi$ is the indicator function of the event in brackets and $T_{I}^{(j)}$ is the $j$-th return time to $I$ with the natural identification between the two auxiliary processes introduced in [AN]. It follows from the formula of the transition kernel with starting point $\Theta$ that

$$
\widetilde{\mathbb{E}}_{\Theta}\left(T_{I}^{(1)}\right)=\int_{I} \mathbb{E}_{\xi}\left(T_{I}^{(1)}\right) \phi(d \xi) \leqq M_{I}
$$

and

$$
\tilde{\mathbb{E}}_{\Theta}\left(T_{I}^{(j+1)}-T_{I}^{(j)}\right)=\int_{I \times\{1\}} \mathbb{E}_{\xi}\left(T_{I}^{(j+1)}-T_{I}^{(j)}\right) \phi(d \xi)=\int \mathbb{E}_{\xi}\left(\mathbb{E}_{x T_{I}^{(j)}}\left(T_{I}\right)\right) \phi(d \xi) \leqq M_{I}
$$

where we exploit the Markov property.

Equation (3.6) follows from the equivalence theorem of [AN].

Now we have the following relation valid for Markov chains with a recurrent state (see e.g. [Ch] Chap. 1, Sect. 10)

$$
\tilde{Q}(\Theta)=\frac{1}{\widetilde{\mathbb{E}}_{\Theta}\left(\widetilde{T}_{\Theta}\right)} .
$$

By using (3.3) of Lemma 3.3, (3.5) and (3.6) we get that

$$
Q(I \times\{1\}) \geqq \frac{1}{M_{I}} \geqq \frac{c}{(\ln E)^{2}}
$$

for $E$ sufficiently small and $I \in \mathscr{I}_{b E, \delta}$ with $c$ constant independent from $E$ and $I$.

We are now in the position to state the main result of this section.

3.4. Theorem. There exists $b>0, E_{0}>0, c>0$ and $n_{0}$ a positive integer such that for $0<E<E_{0}$, and $\eta \in J_{b E} \times\{1\}$ and $n \geqq n_{0}$,

$$
p_{E}^{(n)}(\xi, \eta) \geqq \frac{c}{(\ln E)^{2}} .
$$

Proof. From the exponential approach (3.4) and the bound (3.11) we have for any $\xi \in \mathscr{S}$ and $I \in \mathscr{J}_{b E}$,

$$
P_{E}^{(n)}(\xi, I \times\{1\}) \geqq \frac{c}{(\ln E)^{2}}
$$

for $n$ sufficiently large and a suitable constant $c$. Therefore for $\eta \in J_{b E} \times\{1\}$ we have, by applying the estimate (3.1) of Lemma 3.1, 


$$
p_{E}^{(n+2)}(\xi, \eta)=\int p_{E}^{(n)}(\xi, \zeta) p_{E}^{(2)}(\zeta, \eta) d \zeta \geqq \int_{I \times\{1\}} p_{E}^{(n)}(\xi, \zeta) p_{E}^{(2)}(\zeta, \eta) d \zeta \geqq \frac{c}{(\ln E)^{2}}
$$

with a possibly different constant $c$.

\section{Main Theorem}

We are now in a position to state and prove our main result.

4.1. Theorem. Let $\rho(E)$ be the absolutely continuous part of the density of states for the Hamiltonian defined in (2.1) Then there exist $E_{0}>0$ and $c>0$ such that for $0<|E|<E_{0}$,

$$
\rho(E) \geqq \frac{c}{(\ln |E|)^{4}|E|} .
$$

Proof. We first remark that

$$
q_{E}^{(N)}\left(\Delta_{0}, \Delta\right)=p_{E}^{(N)}\left(\left(\ln \left|\Delta_{0}\right|, \operatorname{sgn} \Delta_{0}\right),(\ln |\Delta|, \operatorname{sgn} \Delta)\right) \frac{1}{|\Delta|},
$$

and therefore it follows from Theorem 3.4 and (2.13) that for $0<E<E_{0}$ and $b$ sufficiently large we have

$$
\rho_{N}(E) \geqq c \int_{b E}^{1 / b E} \frac{1}{(\ln E)^{4}|E-x| x} d x \geqq \frac{c}{(\ln E)^{4}} \int_{b E}^{1 / b E} \frac{1}{x^{2}} d x \geqq \frac{c}{(\ln E)^{4} E},
$$

where the same symbol $c$ denotes different constants.

Now the integrated density of states $k(E)$ is given by

$$
k(E)=\lim _{N \rightarrow \infty} \mathbb{E} k_{N}(E)=\lim _{N \rightarrow \infty} \int_{-\infty}^{E} \rho_{N}(x) d x .
$$

From (4.2) and (4.3) we get that the density of states $\rho(E)$, which is by definition the derivative of $k(E)$ in the full measure set where the derivative exists, satisfies

$$
\rho(E) \geqq \frac{c}{(\ln E)^{4} E}
$$

in some interval $\mathrm{O}<E<E_{0}$. With obvious changes all our arguments work also for negative $E$ 's, so that we finally get (4.1) with some possibly smaller constant $E_{0}$.

\section{Appendix. Proof of Lemmas 3.1, 3.2 and 3.3}

Proof of Lemma 3.2. Let $[\alpha, \beta]$ be an interval contained in the support of the probability density of the potential $f$ such that $\inf _{v \in[\alpha, \beta]} f(v)>0$.

Let $\xi=(u, 1), \eta=(v, 1)$ with $u, v \in I$, and $I \in \mathscr{I}_{b E, \delta}$. In the inequality

$$
p_{E}^{(2)}(\xi, \eta) \geqq \int_{A \times\{-1\}} p_{E}(\xi, \zeta) p_{E}(\zeta, \eta) d \zeta,
$$


we take as $A$ the set

$$
A=\left\{z:\left|z-\frac{\ln \beta^{2}+\ln \alpha^{2}}{2}+u\right|<\frac{\ln \beta^{2}-\ln \alpha^{2}}{8}\right\} .
$$

By using (2.15) that defines the transitions of the Markov chain it is easy to see that this choice of $A$ ensures that for $b$ sufficiently large, $E$ sufficiently small and $\zeta=(z,-1)$ with $z \in A$,

$$
p_{E}(\xi, \zeta) \geqq \inf _{w \in[\alpha, \beta]} \frac{w}{2} g(w) \equiv \gamma>0, \quad \text { and similarly } p_{E}(\zeta, \eta) \geqq \gamma
$$

if $|v-u|<\frac{\ln \beta^{2}-\ln \alpha^{2}}{8}$. Therefore if we take $\delta=\frac{\ln \beta^{2}-\ln \alpha^{2}}{8}$,

$$
p_{E}^{(2)}(\xi, \eta) \geqq \gamma^{2} \delta^{2},
$$

Proof of Lemma 3.2. Let us first take $x^{(i)}=\left(u_{i}, 1\right) \in \mathbb{R} \times\{1\}$. Assume that $u_{i}>\ln E$, then by iterating twice the relation (2.9) (remember that we have reversed the direction of time) we get the relation already exploited in $[E R], x^{(i+2)}=\left(u_{i+2}, \varepsilon_{i+2}\right)$ with

$$
u_{i+2}=u_{i}+\ln \left(\frac{V_{i+1}^{2}}{V_{i}^{2}}\right)+\ln \left(\frac{1-E \exp \left(-u_{i}\right)}{1+\frac{E \exp \left(u_{i}\right)-E^{2}}{V_{i}^{2}}}\right), \quad \varepsilon_{i+2}=1 .
$$

We can write the first formula of (4.10) in the form

$$
u_{i+2}=u_{i}+r_{i}+s_{i}
$$

where $r_{i}$ are bounded i.i.d. random variables with symmetric distribution and $s_{i}$ are non-positive random variables. The decomposition (4.11) will be essential to obtain our result.

By using (4.10) and the fact that the distribution of the variables $V_{i}$ 's has compact support contained in $(0, \infty)$, we see that there exist constant $K_{1}, K_{2}>0$ such that for $E$ sufficiently small if $x^{(i)}=\left(u_{i}, 1\right)$ with $u_{i} \geqq K_{1}-\ln (E)$, then $x^{(i+2)}=\left(u_{i+2}, 1\right)$ with

$$
-K_{2}-\ln (E) \leqq u_{i+2} \leqq K_{2}-\ln (E) \text {. }
$$

Let us define the stopping time

$$
\tau^{(1)}=\min \left\{2 j \mid u_{2 j} \leqq \ln (E)\right\} .
$$

We can prove that if $x^{(0)}=\left(u_{0}, 1\right)$ with $u_{0}>\ln (E)$, then

$$
\mathbb{P}_{x^{(0)}}\left(\tau^{(1)} \geqq c(\ln (E))^{2} \leqq \frac{3}{4},\right.
$$

whre $c$ is a constant independent of $E$. Indeed from (4.12) we can assume $u_{0} \leqq K_{2}-\ln (E)$. Then

$\mathbb{P}_{u_{0}}\left(\tau^{(1)} \geqq c|\ln E|^{2}\right) \leqq \mathbb{P}\left(\sum_{n=1}^{\left[c|\ln E|^{2}\right]} r_{n} \geqq \ln u_{0}-\ln (E)\right) \leqq \mathbb{P}\left(\sum_{n=1}^{\left[c|\ln E|^{2}\right]} r_{n} \geqq K_{2}-2 \ln (E)\right)$, 
where we have used the decomposition (4.11) and in particular the non-positivity of the variables $s_{i}$ 's. For $c$ is sufficiently large the right-hand side of (4.15) is less than $\frac{3}{4}$ by the central limit theorem and this proves (4.14).

Let now $b, \delta, c, E_{0}$ constants such that the statement of Lemma 3.1 is verified, i.e. such that for $0<E<E_{0}, I \in \mathscr{J}_{b, E, \delta}$ and every $\left.\xi, \eta \in I \times\{1\}\right]$

$$
p_{E}^{(2)}(\xi, \eta) \geqq c>0 \text {. }
$$

Let $\bar{I}=\left(y_{1}, y_{2}\right)$ be an interval such that the following properties are verified:

i) $I \subset \bar{I} \subset J_{b E}$;

ii) it is not possible that for $x^{(i)}=\left(u_{i}, 1\right)$ with $u_{i} \geqq y_{2} x^{(i+2)}=\left(u_{i+2}, 1\right)$ with $u_{i+2} \leqq y_{1}$;

iii) the length of $\bar{I}|\bar{I}|$ is bounded uniformly with respect to $E$.

These properties are verified by (4.10) if we take $|\bar{I}| \geqq 4 \ln \bar{\beta}^{2} / \bar{\alpha}^{2}$ if $b$ is sufficiently large and $E$ is sufficiently small.

Let $\bar{\tau}^{(2)}$ be the stopping time

$$
\bar{\tau}^{(2)}=\min \left\{j \geqq 1: x^{(j)} \in \bar{I} \times\{1\}\right\} .
$$

If $x^{(0)}=\left(u_{0}, 1\right)$ with $u_{0}<\ln E$ we have $x^{(1)}=\left(u_{1}, 1\right)$ with $u_{1} \geqq-\ln (b E)$ if $b$ is sufficiently large. Therefore

$$
\begin{aligned}
\mathbb{P}_{x^{(0)}}\left(\bar{\tau}^{(2)} \geqq c|\ln E|^{2}\right) & \leqq \mathbb{P}\left(\sum_{i=1}^{\left[(c / 2)|\ln E|^{2}\right]} r_{i} \geqq u_{1}-\ln (b E)\right) \\
& \leqq \mathbb{P}\left(\sum_{i=1}^{\left[(c / 2)|\ln E|^{2}\right]} r_{i} \geqq \bar{c}|\ln E|\right) \leqq \frac{3}{4},
\end{aligned}
$$

if $c$ is sufficiently large and $\bar{c}$ is a suitable positive constant (we used the properties i), ii) of the interval $\bar{I}$ and, as in the previous estimate, the decomposition (4.11) and the central limit theorem).

We are interested in the stopping time

$$
\tau^{(2)}=\min \left\{j \geqq 1: x^{(j)} \in I \times\{1\}\right\} .
$$

By applying Lemma 3.1, (4.17) and the property iii) of $\bar{I}$ we can show that for $0<E<E_{0}$ with $E_{0}$ sufficiently small for $x^{(0)}=\left(u_{0}, 1\right)$ with $u_{0}<\ln E$,

$$
\mathbb{P}_{x^{(0)}}\left(\tau^{(2)} \geqq c|\ln E|^{2}\right) \leqq 1-p
$$

for some positive $p>0$. Indeed after the process reaches the interval $\bar{I}$ the process can reach $I$ in an even number of steps with probability bounded from below since by (4.17) it can move in two steps in an interval of size $\delta$ with density bounded from below.

After reaching $I \times\{1\}$ by (4.17) the process can return to $I \times\{1\}$ after an even number of steps with positive probability. Summarizing we can find an odd integer $n_{0} \geqq c|\ln E|^{2}$ for some constant $c$ such that

$$
p_{E}^{\left(n_{0}\right)}(\xi, \eta) \geqq c>0 \quad \text { for any } \quad \xi=(u, 1) \text { and } \eta=(v, 1) \text { with } v \in J_{b E} .
$$

The proof is completed by remarking that if $x^{(0)}=\left(u_{0}-1\right)$, then $x^{(1)}=\left(u_{1}, 1\right)$. 
Therefore we have that

$$
p_{E}^{\left(n_{0}+1\right)}(\xi, \eta) \geqq c>0 \quad \text { for any } \xi=(u,-1) \text { and } \eta=(v, 1) \text { with } v \in J_{b E} .
$$

By combining (4.17), (4.20) and (4.21) we obtain that

$p_{E}^{\left(2 n_{0}\right)}(\xi, \eta) \geqq c>0 \quad$ for any $\quad \xi \in \mathscr{S}=\mathbb{R} \times\{-1,1\} \quad$ and $\eta=(v, 1)$ with $v \in J_{b E}$.

Proof of Lemma 3.3. The result follows easily from the proof of Lemma 3.2. Indeed the arguments used there imply that if $I \in \mathscr{I}_{b, E, \delta}$ for appropriate constants $b$ and $\delta$,

$$
\mathbb{P}_{\xi}\left(T_{I} \leqq c(\ln E)^{2}\right) \geqq \bar{p}>0,
$$

where the constants $c$ and $\bar{p}$ are uniform in $\xi \in \mathscr{S}=\mathbb{R} \times\{-1,1\}$ and $E<E_{0}$. Let then $E_{k}$ be the event that $T_{I}>k c(\ln E)^{2}$. From (4.23) we have that

$$
\mathbb{P}_{\xi}\left(E_{1}\right) \leqq 1-\bar{p} \text {. }
$$

By applying the Markov property we get

$$
\mathbb{P}_{\xi}\left(E_{k+1}\right)=\mathbb{P}_{\xi}\left(E_{k} \cap E_{k+1}\right) \leqq \mathbb{P}_{\xi}\left(E_{k}\right) \sup _{\eta} \mathbb{P}_{\eta}\left(E_{1}\right)
$$

Therefore

$$
\mathbb{P}_{\xi}\left(E_{k}\right) \leqq(1-\bar{p})^{k}
$$

uniformly in $\xi \in \mathscr{S}$. But then

$$
\begin{aligned}
\mathbb{E}_{\xi}\left(T_{I}\right) & \leqq c(\ln E)^{2}\left(1-\mathbb{P}_{\xi}\left(E_{1}\right)\right)+\sum_{k=1}^{\infty}(k+1) c(\ln E)^{2}\left(\mathbb{P}_{\xi}\left(E_{k}\right)-\mathbb{P}_{\xi}\left(E_{k+1}\right)\right) \\
& =c(\ln E)^{2}\left(1+\sum_{k=1}^{\infty} \mathbb{P}_{\xi}\left(E_{k}\right)\right) \leqq c(\ln E)^{2}\left(1+\sum_{k=1}^{\infty}(1-\bar{p})^{k}\right)=\frac{c(\ln E)^{2}}{\bar{p}}
\end{aligned}
$$

\section{References}

[AN] Athreya, K. B., Ney, P.: A new approach to the limit theory of recurrent Markov chains. Trans. Am. Math. Soc. 245, 493 (1978)

[BCKP] Bovier, A., Campanino, M., Klein, A., Perez, J. F.: Smoothness of the density of states in the Anderson model at high disorder. Commun. Math. Phys. 114, 439-462 (1988)

[CK] Campanino, M., Klein, A.: A supersymmetric transfer matrix and differentiability of the density of states in the one-dimensional Anderson model. Commun. Math. Phys. 104, 227-241 (1986)

[C] Chung, K. L.: Markov chains with stationary transition probabilities. Berlin, Heidelberg, New York: Springer 1960

[D] Doob, J. I.: Stochastic processes. New York: John Wiley 1953

[Dy] Dyson, F. J.: The dynamics of a disordered linear chain. Phys. Rev. 92, 1331-1338 (1953)

[ER] Eggarter, T. P., Riedinger, R.: Singular behavior of tight chains with off-diagonal disorder. Phys. Rev. B18, 569-575 (1978)

[ST] Simon, B., Taylor, M.: Harmonic analysis on $S L(2, \mathbb{R})$ and smoothness of the density of states in the one-dimensional Anderson model. Commun. Math. Phys. 101, 1-19 (1985) 
[S] Spencer, T.: The Schrödinger equation with a random potential. A mathematical review. In: Critical Phenomena, Random Systems and Gauge Theory. Osterwalder, K., Stora, R. (eds.). Amsterdam: Elsevier 1986

[TC] Theodorou, G., Cohen, M. H.: Extended states in a one-dimensional system with off-diagonal disorder. Phys. Rev. B13, 4597 (1976)

[W] Wegner, F.: Bounds on the density of states in disordered systems. Z. Phys. B44, 9-15 (1981)

Communicated by T. Spencer

Received September 19, 1988 